\title{
Influence of Variation in Eumelanin Content on Absorbance Spectra of Liquid Skin-
}

\section{like Phantoms}

\author{
Jacoba E Smit* ${ }^{* 1,2}$, Anne F Grobler ${ }^{3}$, Raymond W Sparrow ${ }^{4}$ \\ ${ }^{1}$ Biophotonics group, National Laser Centre, CSIR, PO Box 395, Pretoria, 0001, South Africa \\ ${ }^{2}$ Structural Biology, Biosciences, CSIR, PO Box 395, Pretoria, 0001, South Africa \\ ${ }^{3}$ Unit for Drug Research and Development, North-West University, Potchefstroom, South \\ Africa \\ ${ }^{4}$ Synthetic Biology ERA, Biosciences, CSIR, PO Box 395, Pretoria, 0001, South Africa
}

*Corresponding author's name and email: Jacoba E Smit (KSmit@csir.co.za)

\footnotetext{
${ }^{*}$ To whom correspondence should be addressed, at Structural Biology, Biosciences, CSIR, PO Box 395, Pretoria, 0001, South Africa; fax: + 2712841 3651; email: KSmit@ csir.co.za;
} 


\section{ABSTRACT}

The attenuation behaviour of two different types of skin-like phantoms representing the range of Skin Types I to VI were investigated and compared to real human skin. Intralipid and Pheroid $^{\mathrm{TM}}$ artificial lipid membrane vesicles respectively were added to synthetic eumelanin concentrations ranging from 0.0044 to $0.13 \mathrm{mg} / \mathrm{mL}$ to produce skin-like phantoms. Spectrophotometric absorbance and transmittance measurements were performed. Results predicted some of the non-monotonic trends observed in real human skin, albeit shifted more towards the visible wavelength range. There exist however an underlying difference in interaction between the melanin and the Pheroid ${ }^{\mathrm{TM}}$ and Intralipid skin-like phantoms. 


\section{INTRODUCTION}

Minimalistic or non-invasive diagnosis and treatment of skin disorders with light requires accurate information regarding the optical properties of skin (1), as well as knowledge of the propagation of light from an incident light source in biological tissue (2-5). The range of photon events characterising light-biomatter interaction includes absorption, transmission, auto-fluorescence, scattering and Raman scattering. Spectrophotometric (SP) and Integrating Sphere (IS) methods are commonly used to extract information regarding absorption and scattering properties of samples, although in the field of skin optics the latter method is used more widely (see 1,6). However, since the scattering components are the tissue microstructures of in vivo skin tissue, reliable results of in vitro IS measurements are difficult to obtain (5). Wavelength dependent absorption and scattering of various tissues, including bulk skin tissue and selected skin cell tissue components, have been measured in vitro $(5,7-$ 11) and in vivo (12-15) using various measuring methods.

Computational light propagation models aid in enhancing the understanding of lightbiomatter interactions. It affords the opportunity to investigate the underlying principles governing light-biomatter interactions though the control and variation of specific parameter values (Fig. 1(b)). This is not always possible, feasible or possibly too time consuming with in vitro and in vivo experiments. Human skin tissue is too complex and dynamic to be adequately simulated with the currently available computational light propagation models, especially since these models only take the macroscopic structure of skin tissue in account (see 2,5,16). Healthy human skin tissue consists of different layers having different cell types, such as keratinocytes, fibroblasts and melanocytes and other components like keratin, elastin, collagen fibres, arteries, sweat glands, etc ( and Fig. 1(a))17). Ultimately the accuracy of a light propagation models depends on a thorough knowledge of the optical properties of the 
cell tissue microstructure $(7,18)$, for example the effect that skin pigments have on absorption and scattering of incident light.

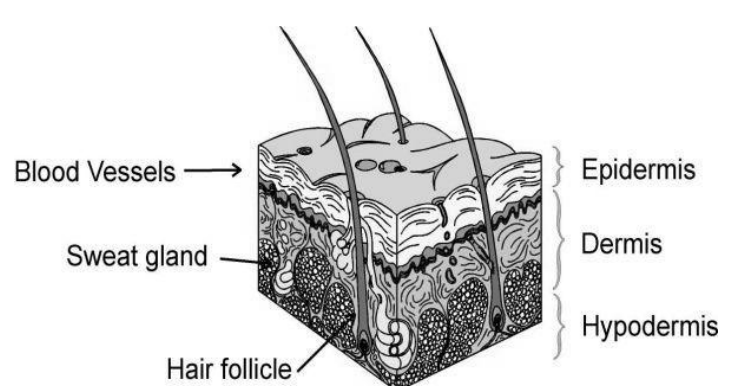

(a)

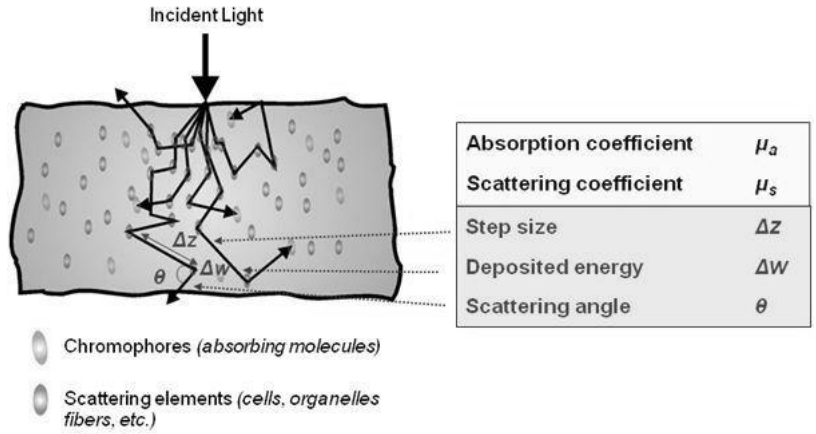

(b)

Fig. 1: (a) Schematic representation of human skin. (b) Skin model showing the different skin layers. Graphics reproduced with the permission of Dr. J.S. Dam (http://cv.js-

dam.dk/docs/pubs/Dam2000phd_Optical\%20analysis\%20of\%20biological\%20media. $p d f)$.

$<$ Figure 1>

Skin pigments include melanin, haemoglobin, bilirubin and carotene, with melanin and haemoglobin (oxy-haemoglobin and deoxy-haemoglobin) the main contributors to skin tone (phototype). The melanin responsible for the different skin phototypes is located in the thin epidermal layer of the skin, while haemoglobin dominates the absorption properties of the dermis $(9,19)$. Knowledge of the absorption of light in the epidermal layer is for example crucial in the planning of the therapeutic treatment parameters (laser power and treatment time) of skin tumours situated in the dermis $(20,21)$. Darker skin phototypes contains up to twice the melanin content of paler skin phototypes and may be less susceptible to the effects of skin cancer development due to the presence of more darker photoprotective melanin (22). Most of the studies conducted to date however have focussed on Skin Types I and II (light 
skin phototypes), with only a few studies recorded for Skin Types III to VI (darker skin phototypes) (see 23,24). Despite extensive investigations on both natural and synthetic melanin, proper characterisation of the melanin complex and the role its composition play in skin colour still eludes us today.

South African skin phototype demographics afford the ideal niche opportunity to conduct studies to establish a solid base for the optical characterisation of melanin in different skin phototypes. Large quantities of skin samples of all skin phototypes however are often always limited. Hence the use of skin-like phantoms. Bashkatov et al. (25) measured the optical properties of gelatin skin-like phantoms prepared from different concentrations of synthetic and natural (from Sepia officianalis) melanin. Fat emulsions like Intralipid are commonly used to mimic light propagation in turbit media (26) and hence Shimada et al. (27) used similar gelatin and Sepia melanin phantoms and added Intralipid to mimic the scattering properties of human skin.

The purpose of this study is to (a) extract and compare attenuation coefficients of different liquid skin-like phantoms representing Skin Types I to VI with Spectrophotometry and (b) determine how well these phantoms can predict wavelength dependent trends observed in real human skin. It falls within the larger objective of knowledge acquisition about the optical properties of skin cell tissue to enhance the accuracy of 3D skin our computational models representing Skin Types I to VI. Two different skin-like phantom sets were investigated. The first set was prepared from different concentrations of synthetic eumelanin and added Intralipid (20\% fat emulsion), while the second was prepared from the same concentrations of the eumelanin but with added bilipid membrane artificial vesicles (Pheroid $^{\mathrm{TM}}$ ) recently developed by the Unit for drug development and research, Potchefstroom Campus, North-West University in South Africa. Even though the vesicles do not contain all the microstructures found in skin tissue cells, similar to all other cell tissue 
components they are bounded by a bilipid membrane (28). To establish a more physiological standard against which to compare the optical properties of melanocytes and keratinocytes, the optical characterisation of the Pheroid ${ }^{\mathrm{TM}}$ vesicles was used to determine the contribution of lipid membranes to the overall optical properties of the skin tissue components.

\section{MATERIALS AND METHODS}

Sample preparation. A melanin aqueous stock solution at $\mathrm{pH} \sim 11.0$ was prepared by dissolving synthetic eumelanin (Sigma-Aldrich M0418) in distilled water through addition of $\mathrm{NaOH}$ (29). To aid comparison with real human skin results, the $\mathrm{pH}$ of half of this stock solution was then lowered to physiological $\mathrm{pH}(\mathrm{pH} \sim 7.01)$ by adding $\mathrm{HCl}$. From these two stock solutions melanin samples at various concentrations (from $0.0088 \mathrm{mg} / \mathrm{mL}$ to $0.13 \mathrm{mg} / \mathrm{mL}$ ) were prepared. Skin-like phantoms were prepared by adding either $3 \mu \mathrm{L}$ or 30 $\mu \mathrm{L}$ Pheroid $^{\mathrm{TM}}$ or Intralipid (Sigma-Aldrich I141) respectively to $3 \mathrm{~mL}$ of each of the $\mathrm{pH} \sim$ 7.01 melanin samples. The Intralipid (IL) is a phospholipid-stabilized soybean oil, supplied as $20 \%$ fat emulsion. The Pheroid ${ }^{\mathrm{TM}}$ is an micro-emulsion containing $3.8 \%$ lipid content consisting mainly of ethyl esters of the fatty acids obtained from soybean oil (30).

Spectrophotometry. Absorbance and transmittance spectra of the samples were measured with a UV-VIS spectrophotometer (Shimazdu UV-1650 PC) using standard $1 \mathrm{~cm}$ pathlength cuvettes over the wavelength range 370 to $900 \mathrm{~nm}$. The attenuation coefficient $\left(\mu_{t} \lambda\right)$ is the decrease in the light intensity through the sample and is equal to the combination of the 
absorption and scattering coefficients of the sample (31). Attenuation coefficients were calculated from

$$
\mu_{t} \lambda=\frac{-\ln T \lambda}{L} \quad 1 / \mathrm{cm}
$$

with $T \lambda$ the wavelength dependent transmittance and $L$ the pathlength (in $\mathrm{cm}$ ). Alternatively, since the relation between absorbance and transmittance is given by $A \lambda=-\log T \lambda$, the attenuation coefficients could be calculated from a modified version of the Beer-Lambert law

$$
\mu_{t} \lambda=A \lambda \ln 10 / L \quad 1 / c m
$$

where $A(\lambda)$ is the wavelength dependent absorbance of the sample and $L$ the pathlength $(\mathrm{cm})$.

\section{RESULTS AND DISCUSSION}

\section{Absorbance spectra of melanin only samples}

Attenuation coefficients $\left(\mu_{t} \lambda\right)$ for the melanin only samples at $\mathrm{pH} \sim 7.01$ were calculated from the absorbance spectra. The spectrophotometer used cannot measure reflectance spectra for liquid samples, and hence we were unable to resolve the total attenuation coefficient into its absorption and scattering components. Even though the attenuation coefficient results mainly displayed absorption coefficient trends as observed by other research groups and we therefore could have made the assumption that our melanin only samples had very little 
scattering, we will refrain from making such an assumption and only report our results as attenuation behaviour.
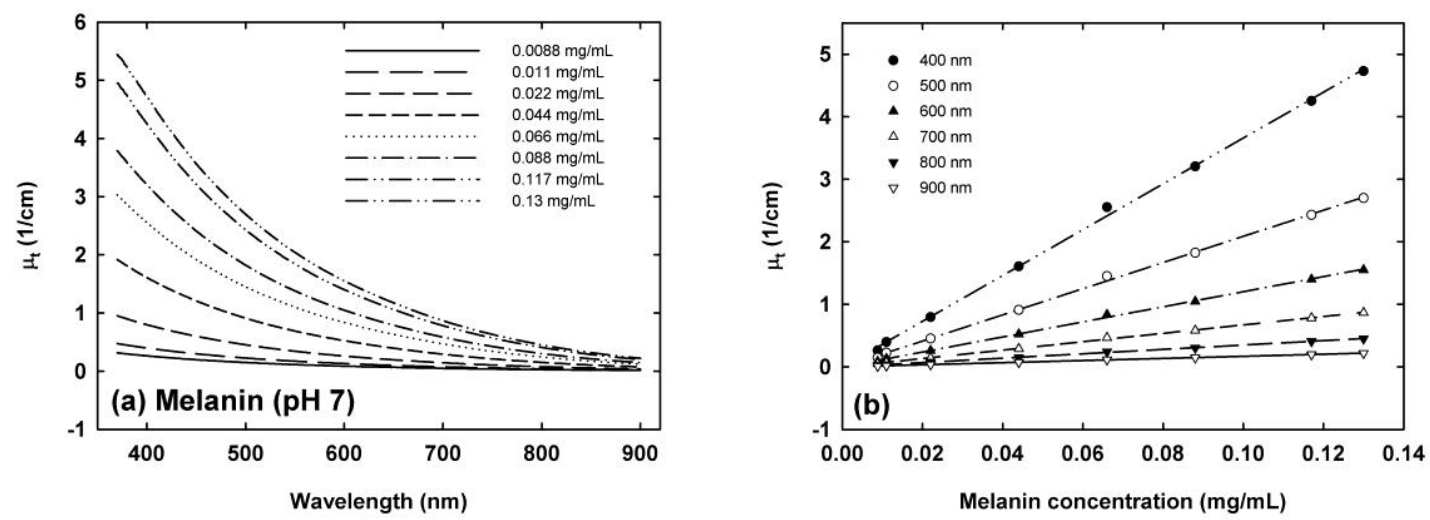

Fig. 2: Attenuation coefficient as a function of (a) wavelength and (b) melanin concentration for synthetic eumelanin samples at $p H \sim 7.01$.

$<$ Figure 2>

Resultant spectra displayed broadband attenuation without any chromophoric peaks (Fig. 2(a)), increasing monotonically towards shorter wavelengths for all measured concentrations. Spectra for samples at $\mathrm{pH} \sim 11.00$ (not shown) were similar to the spectra of the $\mathrm{pH} \sim 7.01$ samples. The monotonic increase with decreasing wavelength is similar to previously observed results for synthetic melanin (see for example 29, and 32).

Figure 2(b) shows attenuation coefficients as a function of melanin concentration at different wavelengths. Spectra followed the Beer-Lambert law's linearity (Fig. 2(b)), indication that the monotonic behaviour can be described by exponential decay curves. 


\section{Absorbance spectra of skin-like phantoms}

In an article on human skin chromophores, Young (19) referred to the use of diffuse in vivo reflectance spectroscopy to determine the contribution of epidermal melanin to the apparent absorbance spectra of skin as employed by Kollias and Baqer (33). The technique involves subtracting the absorbance spectrum of vitiligo skin, i.e. skin without or containing very little melanin, from the spectrum of normal skin in the same individual. Work performed by Jacques and McAuliffe (34) on determining the internal absorption coefficients of human melanosomes generated similar absorbance spectra as the diffuse reflectance spectra, hence supporting the use of the latter technique. In the present study the Pheroid ${ }^{\mathrm{TM}}$ and Intralipid were used respectively to represent the lipid membrane contribution of skin cells and the assumption was made that vitiligo skin could be represented by skin-like phantoms consisting of either $0.1 \% \mathrm{v} / \mathrm{v}$ or $1.0 \% \mathrm{v} / \mathrm{v}$ (i.e. either $3 \mu \mathrm{L}$ or $30 \mu \mathrm{L}$ in $3 \mathrm{~mL}$ distilled water) Pheroid ${ }^{\mathrm{TM}}$ or Intralipid solutions only. Spectra of these "vitiligo" skin-like phantoms were thus subtracted from the melanin-containing skin-like phantom samples to determine the melanin contribution of the phantoms, analogous to the Kollias and Baqer (33) technique (see Figs. $3(a)-(d))$. 

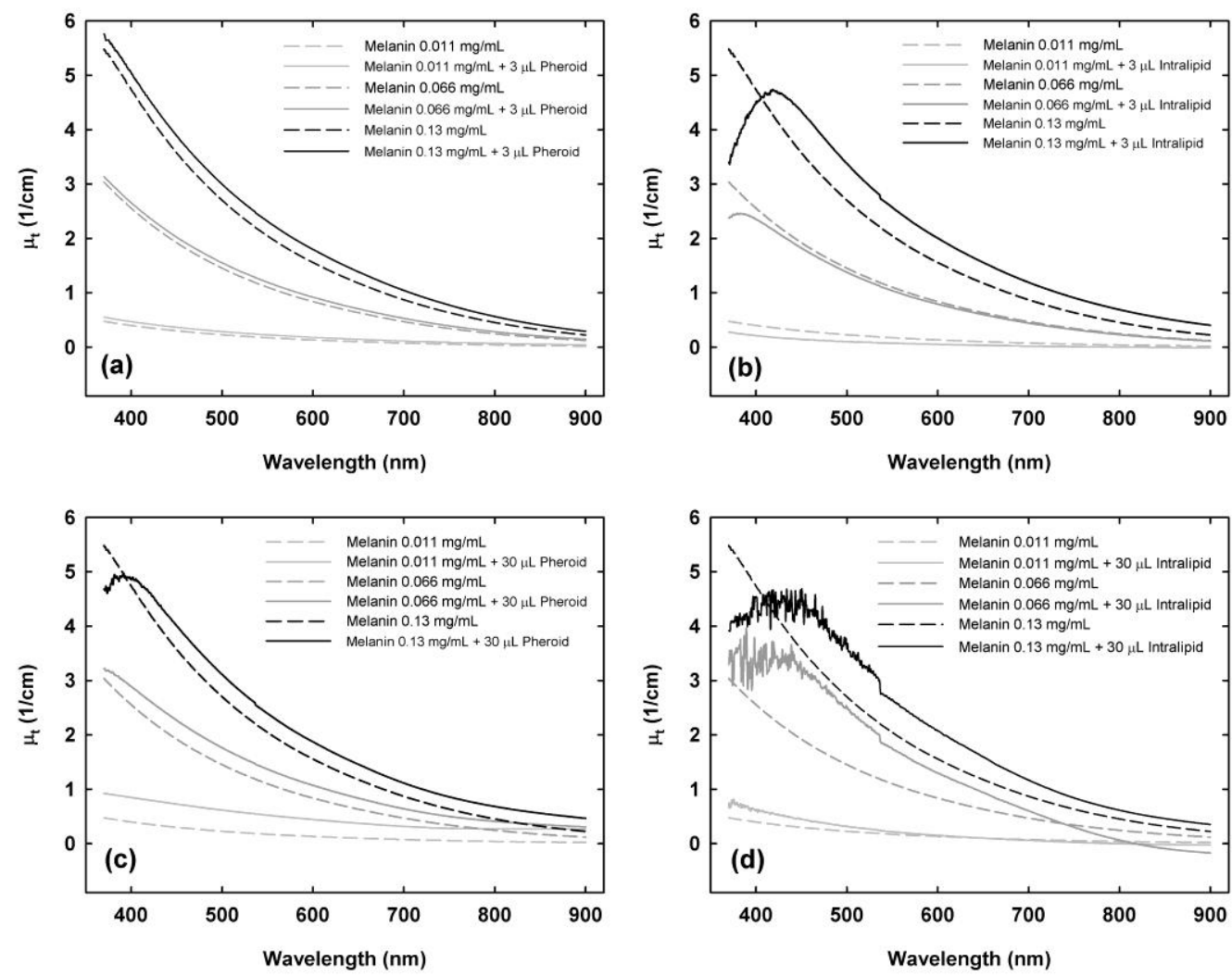

Fig. 3: Attenuation coefficient as a function of wavelength for skin-like phantoms containing (a) $3 \mu$ Pheroid $^{T M}$, (b) $3 \mu$ L Intralipid, (c) $30 \mu$ L Pheroid $^{T M}$ and (d) $30 \mu L$ Intralipid. Phantoms are compared to the melanin only samples of same concentrations from Fig. 2(a).

$<$ Figure 3>

Figures 3(a)-(d) shows attenuation coefficients for three different melanin concentrations (representing light, medium-dark and dark skin phototypes, or alternatively, to a melanin concentration increase in skin exposed to increasing amounts of UV radiation) for the different skin-like phantoms compared to the melanin only samples of the same concentrations. The phantoms prepared from the $3 \mu \mathrm{L}$ Pheroid $^{\mathrm{TM}}$ or Intralipid (Figs. 3(a) and (b)) represent skin having thinner stratum corneum and epidermis layers (i.e. a lesser amount 
of lipid membranes), while the phantoms prepared from the $30 \mu \mathrm{L}$ Pheroid ${ }^{\mathrm{TM}}$ or Intralipid (Figs. 3(c) and (d)) represent skin with thicker layers.

Addition of the Pheroid ${ }^{\mathrm{TM}}$ and Intralipid resulted in skin-like phantoms following similar non-monotonic absorbance trends towards shorter wavelengths as observed in real human skin, albeit more towards the visible wavelengths than the observed UVA region in real skin $(12,13,19,35,36)$. Similar spectra have also been observed for human hair eumelanin and Sepia eumelanin (37). Transmittance spectra (not shown) were similar to measured transmittance curves for Caucasian and Dark Black skins, while the resultant calculated attenuation coefficient spectra displayed the more pronounced non-monotonic absorbance behaviour for darker compared to lighter skin phototypes (compare for example with 36).

Meinhardt et al. $(12,13)$ reported non-monotonic behaviour with (a) an increase in skin melanin concentration, i.e. higher skin phototype, (b) increased UV exposure of the dorsal aspect of the forearm compared to the lesser exposed volar aspect, and (c) increased thickness in horny layer (stratum corneum) of the thenar compared to thinner layers of the volar and dorsal aspects of the forearm. Both (b) and (c) are applicable to human subjects having the same skin phototype. The non-monotonicity in the UV exposed skin is attributed to various degrees of UV adaptation including thickening of the stratum corneum and an increase in melanin production, while (c) is attributed to the higher keratin concentration of the thenar's stratum corneum.

Our phantoms displayed similar trends, although more towards the visible region compared to the UV region as in real human skin. Irrespective of the amount of Pheroid ${ }^{\mathrm{TM}}$ or Intralipid added, an increase in melanin concentration led to increases in both non-monotonic behaviour and attenuation towards wavelengths shorter than $550 \mathrm{~nm}$ (compare Figs. 3(a) to (d)). Attenuation behaviour at longer wavelengths still displayed the same monotonic behaviour found in the melanin only samples. 
The increases in non-monotonic behaviour were also more pronounced in samples containing higher amounts of Pheroid ${ }^{\mathrm{TM}}$ or Intralipid. This is similar to results from Meinhardt et al. (13) who observed more pronounced non-monotonic behaviour for measurements made at the thicker skinned thenar region compared to the volar and dorsal aspects of the forearm (refer to Figs. 5(a) and (b) in (13)). However, their thenar results shows higher absorption coefficient values compared to the rest of the thinner skinned forearm. This trend was not so apparent in our results; compare for example Figs. 3(b) and (d). Samples containing melanin concentrations lower than $0.066 \mathrm{mg} / \mathrm{mL}$ did comply with this trend, but the increase in attenuation was not so pronounced as in the Meinhardt et al. study. However, with higher melanin concentration attenuation values of samples containing $30 \mu \mathrm{L}$ Intralipid were lower than corresponding samples containing only $3 \mu \mathrm{L}$ Intralipid, predicting that in darker skin phototypes, areas on the body having thicker skin layers are not necessarily more protected against sun exposure than areas of thinner skin layers. The Meinhardt et al. study was performed on individuals with lighter skin phototypes, and no results were found for similar skin thickness studies performed on dark skin phototypes. It is therefore currently not possible to confirm or discard our predictions against real human skin of darker phototypes.

\section{Pheroid $^{\mathrm{TM}}$ versus Intralipid}

At a first glance, it seems that there is not much difference in attenuation behaviour of skinlike phantoms prepared from Pheroid ${ }^{\mathrm{TM}}$ and Intralipid respectively. However, the Pheroid ${ }^{\mathrm{TM}}$ are spherically shaped bilipid membrane nano-structured artificial vesicles of $500-1000 \mathrm{~nm}$ in diameter, while Intralipid consists of an emulsion of bilipid membrane fragments of 
various sizes, suspended in water. Intralipid is commonly used to mimic the scattering properties of human skin, while this study is the first to use Pheriod ${ }^{\mathrm{TM}}$ as a possible skin tissue simulating phantom.

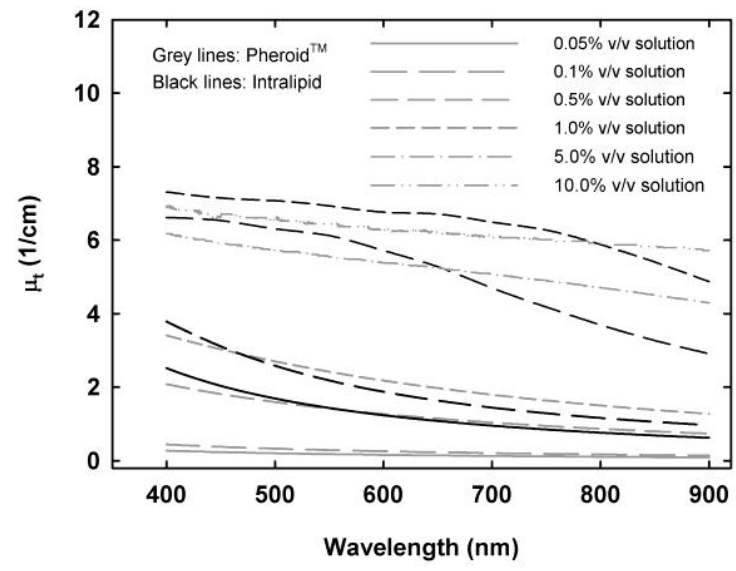

Fig. 4. Comparison attenuation spectra for increasing concentrations of Pheroid ${ }^{T M}$ (grey lines) and Intralipid (black lines).

$<$ Figure 4>

Attenuation coefficients were calculated for different concentrations of the Pheroid ${ }^{\mathrm{TM}}$ and Intralipid respectively. Samples were made up in increasing concentrations of $1.5 \mu \mathrm{L}$ $(0.05 \% \mathrm{v} / \mathrm{v}), 3.0 \mu \mathrm{L}(0.1 \% \mathrm{v} / \mathrm{v}), 15 \mu \mathrm{L}(0.5 \% \mathrm{v} / \mathrm{v})$ and $30 \mu \mathrm{L}(1.0 \% \mathrm{v} / \mathrm{v})$ added to $3 \mathrm{~mL}$ distilled water, while a $150 \mu \mathrm{L}(5.0 \% \mathrm{v} / \mathrm{v})$ and $300 \mu \mathrm{L}(10.0 \% \mathrm{v} / \mathrm{v})$ added to $3 \mathrm{~mL}$ distilled water were made up additionally for the Pheroid ${ }^{\mathrm{TM}}$. Comparison attenuation spectra are shown in Fig. 4. Pheroid ${ }^{\mathrm{TM}}$ spectra displayed broadband absorbance, increasing monotonically towards shorter wavelengths for all concentrations up to $1.0 \% \mathrm{v} / \mathrm{v}$. The 5.0 and $10 \% \mathrm{v} / \mathrm{v}$ concentrations started to show some non-monotonic behaviour. Similarly, only low concentrations of the Intralipid displayed a similar monotonic increasing trend with decreasing wavelength. The higher concentration Intralipid samples also displayed an 
increase in attenuation towards shorter wavelengths, but the behaviour is non-monotonic and tapered off to almost a plateau.

The observed Intralipid non-monotonic attenuation behaviour is a result of its low absorption and high reduced scattering in the visible region $(38,39)$. Higher concentration samples contain greater numbers of unorganised, non-uniform membrane fragments, with a resultant increase in scattering behaviour. No previous studies have been performed regarding the absorption and reduced scattering of the Pheroid ${ }^{\mathrm{TM}}$. By comparing the 5.0 and $10 \% \mathrm{v} / \mathrm{v}$ Pheroid $^{\mathrm{TM}}$ solutions to the 0.5 and $1.0 \% \mathrm{v} / \mathrm{v}$ Intralipid solutions, it appeared that the Pheroid $^{\mathrm{TM}}$ may have a larger reduced scattering than absorption component, but not nearly as large as that of the Intralipid. Most definitely their organised, uniform spherical shapes create less scatter. This will however have to be verified by performing Integrating Sphere measurements on the Pheroid ${ }^{\mathrm{TM}}$ samples.
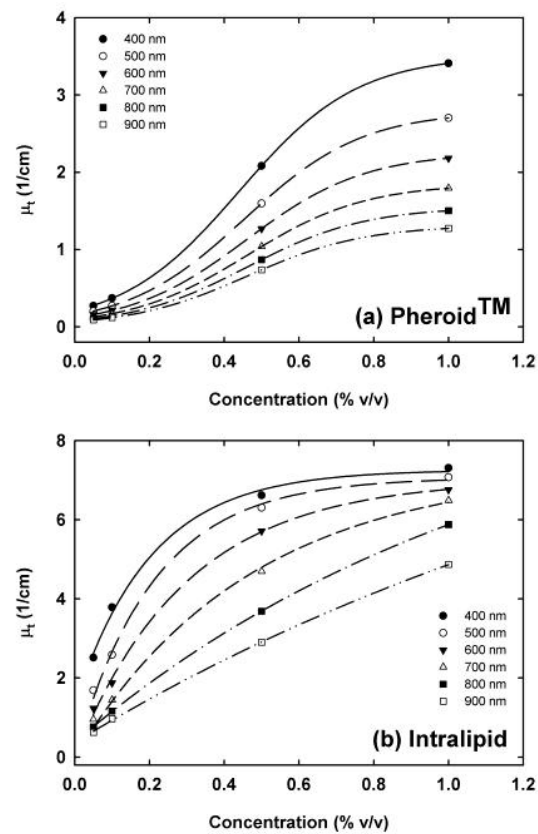

Figure 5. Attenuation coefficient as a function of (a) Pheroid $^{T M}$ and (b) Intralipid concentration over the wavelength range 400 to $900 \mathrm{~nm}$. 
$<$ Figure 5>

Plots of attenuation coefficient as a function of Pheroid ${ }^{\mathrm{TM}}$ (Fig. 5(a)) and Intralipid (Fig. 5(b)) concentration for different wavelengths show that neither phantom obeys the Beer-Lambert law. The Pheroid ${ }^{\mathrm{TM}}$ results could be fitted with Sigmoidal curves over the entire wavelength range. The Intralipid results could not be fitted with a single curve type over the entire wavelength range. Rather, shorter wavelength results could be fitted with "rising exponential curve with a maximum" functions, while the longer wavelength results required straight line curve fitting. This may be due to the differences in structural organization between the Pheroid ${ }^{\mathrm{TM}}$ and Intralipid, but this needs to be investigated in more depth. It must be noted however that different batches of Intralipid differ slightly from one another in their make-up and hence the Intralipid results could differ between batches $(38,39)$.

\section{Melanin interaction with Pheroid $^{\mathrm{TM}}$ and Intralipid}

We investigated the possibility of a difference in interaction between the melanin molecular complex and the lipid membrane structures of the Pheroid ${ }^{\mathrm{TM}}$ and Intralipid. We hypothesised that the non-monotonic behaviour observed in the higher melanin concentration samples (>0.044 ml/mL melanin as seen in Fig. 3) could be attributed to such interaction differences. If the melanin and the lipid membrane structures did not interact and the melanin only samples were used as references/blanks, the resultant attenuation spectra would be the same as the Pheroid ${ }^{\mathrm{TM}}$ or Intralipid only spectra (from Fig. 4). If interactions occur however, the resultant attenuation spectra would reflect this. 
The same skin-like phantom samples as previously (see Fig. 3) were used. However, previously either the Pheroid ${ }^{\mathrm{TM}}$ or Intralipid only phantoms were used as references/blanks, but for this experiment the corresponding melanin only samples (from Fig. 2) were used as references/blanks. For example, if an $0.04 \mathrm{mg} / \mathrm{mL}$ melanin with added Pheroid ${ }^{\mathrm{TM}}$ sample were used, an $0.04 \mathrm{mg} / \mathrm{mL}$ melanin only sample were used as reference/blank, and so forth.
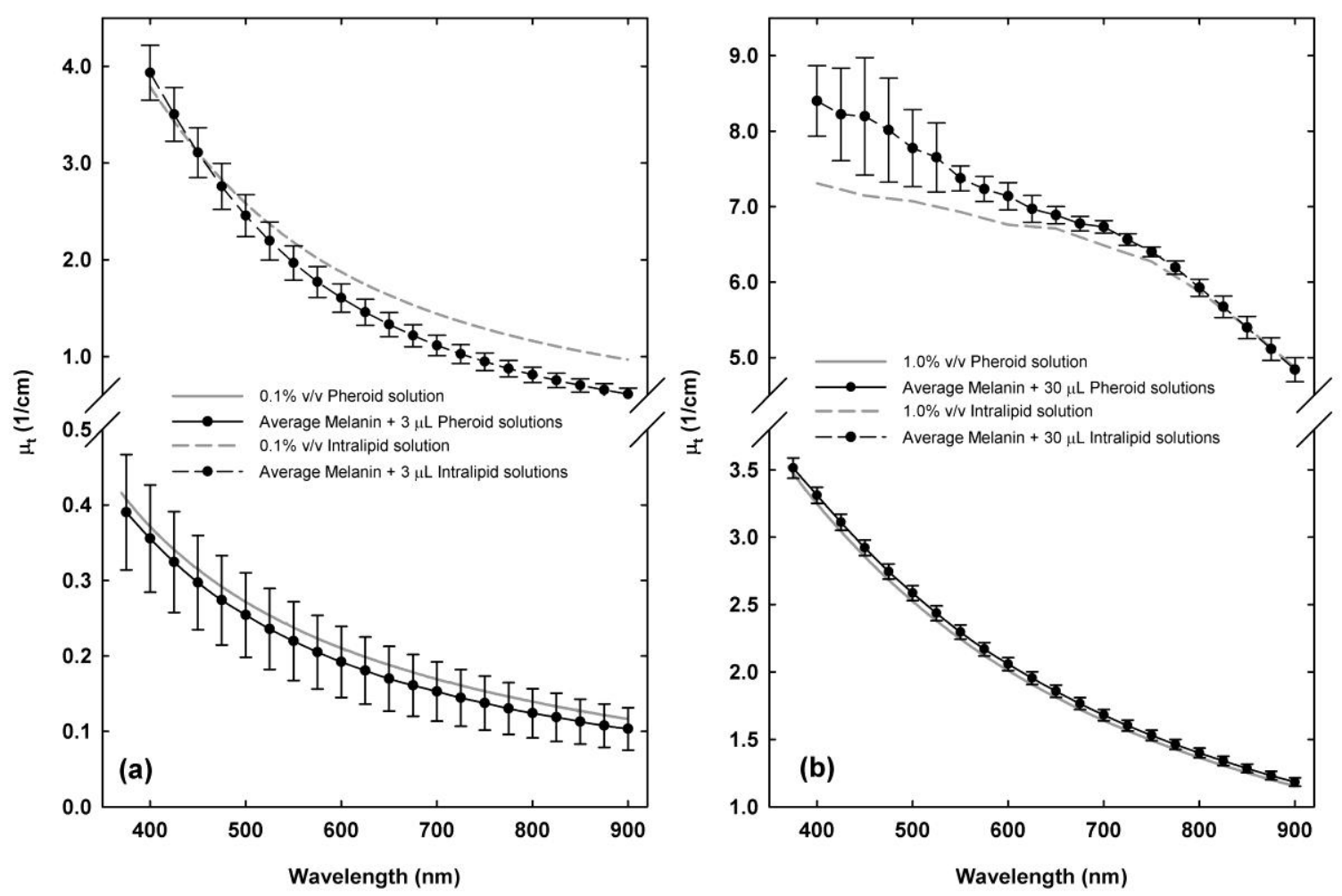

Figure 6. Average of skin-like phantoms made up of 0.0088 to $0.044 \mathrm{mg} / \mathrm{mL}$ melanin concentrations with added (a) $3 \mu L$ and (b) $30 \mu L$ Pheroid $^{T M}$ and Intralipid respectively. Corresponding melanin only concentrations were used as references/blanks. Error bars represent the standard deviation to the average.

$<$ Figure 6>

Attenuation spectra not displaying non-monotonic behaviour was averaged together and their standard deviations calculated (Fig. 6). These average spectra were then compared 
against the Pheroid ${ }^{\mathrm{TM}}$ or Intralipid only spectra from Fig. 4. Fig. 6(a) shows the results for samples having $3.0 \mu \mathrm{L}(0.1 \% \mathrm{v} / \mathrm{v})$ Pheroid $^{\mathrm{TM}}$ or Intralipid added, while Fig. 6(b) show results for added $30 \mu \mathrm{L}(1.0 \% \mathrm{v} / \mathrm{v})$ Pheroid $^{\mathrm{TM}}$ or Intralipid. Spectra from all samples of melanin concentrations $0.044 \mathrm{mg} / \mathrm{mL}$ and lower could be averaged. Irrespective of the amount of Pheroid ${ }^{\mathrm{TM}}$ added, the averaged spectra closely resembled the Pheroid $^{\mathrm{TM}}$ alone spectra. In contrast, for samples with added Intralipid, the averaged spectra diverged from the Intralipid alone spectra. This divergence was also dependent on the amount of added Intralipid and shifted towards shorter wavelengths as the added amount of Intralipid increased. A further observation is that in the case of the added $3.0 \mu \mathrm{L}(0.1 \% \mathrm{v} / \mathrm{v})$ Pheroid $^{\mathrm{TM}}$ or Intralipid, the average spectra underestimated the Pheroid ${ }^{\mathrm{TM}}$ or Intralipid only spectra, while in the case of the added $30 \mu \mathrm{L}(1.0 \% \mathrm{v} / \mathrm{v})$ Pheroid $^{\mathrm{TM}}$ or Intralipid, the opposite occurred.

Spectra of samples with melanin concentrations higher than $0.044 \mathrm{mg} / \mathrm{mL}$ displayed similar non-monotonic behaviour observed when the Pheroid ${ }^{\mathrm{TM}}$ or Intralipid were used as references/blanks (see Fig. 3). Thus there is a clear difference in the interaction of the melanin complex and the lipid membrane structures between high concentration and lower concentration melanin samples, i.e. between darker and lighter skin phototypes.

Furthermore, the type of skin tissue simulating phantom used is important. The results from Figs. 4 to 6 clearly show that even though on a macroscopic level different phantoms appear similar in their predictions, the underlying melanin and lipid membrane interaction can differ significantly, especially for samples representing lighter skin phototypes. It appears that the melanin binds more readily with the lipid membrane fragments found in the Intralipid than with the spherically shaped Pheroid ${ }^{\mathrm{TM}}$. 
Melanin is a family of polymorphous multifunctional biopolymers and its protective role lays in the fact that it binds to cations, anions, drugs and chemicals. (40). The Intralipid lipid membrane fragments are smaller than the Pheroid ${ }^{\mathrm{TM}}$ vesicles; thus making it probably easier for the melanin to bind to the former than to the latter. The melanin sub-complexes also tend to aggregate into increasingly larger particles as the concentration is increased. It is therefore a possibility that such larger aggregates bind to lipid structures more readily than the smaller melanin particles associated with lower melanin concentrations. Our results may therefore predict that photoprotection in darker skin phototypes may not be only a function of epidermal melanin concentration, but that the interaction between the melanin complex and the lipid membranes found in the skin cells may play an equally important role.

We also tried to establish a more physiological standard against which to compare the optical properties of melanosomes and keratinocytes with the use of the Pheroid ${ }^{\mathrm{TM}}$. This required incorporation of the melanin into the Pheroid ${ }^{\mathrm{TM}}$, since the latter can be used to represent skin cells devoid of all internal structure. The results presented are inconclusive as to whether the melanin did indeed incorporate or not. One possible way to establish this is by comparing the melanin-Pheroid ${ }^{\mathrm{TM}}$ phantoms to melanosomes from different skin phototypes. We did not have access to human skin melanosomes and thus such a comparison could not be made. To verify the true amount to which these phantoms represent real skin, such a comparison is required. 


\section{CONCLUSIONS}

The attenuation behaviour of two different types of skin-like phantoms representing the range of Skin Types I to VI were investigated. Results indicated that the phantoms may predict some of the non-monotonic optical characteristics of real human skin and hence suggest the possibility that synthetic eumelanin may be more similar to epidermal eumelanin than previously thought. A comparative study of the Pheroid ${ }^{\mathrm{TM}}$ and Intralipid skin-like phantoms however, revealed an underlying difference in interaction between the melanin and the different phantoms, as well as a difference in the interaction between low and high concentration melanin samples with the constituting lipid particles.

Meinhardt et al. (13) suggested that the non-monotonic absorbance UV behaviour are due to the keratin present in the upper skin layers. Our current skin-like phantoms do not contain keratin. More realistic phantoms can hence be created by addition of keratin. Also, a comparison between the Pheroid ${ }^{\mathrm{TM}}$ phantoms and melanosomes from different skin phototypes could not be made. This will be addressed in the next phase of the study.

Acknowledgements - This work has been supported by CSIR Parliamentary Grants.

\section{REFERENCES}

1. Wilson, B. C. and S. L. Jacques (1990) Optical reflectance and transmittance of tissues: Principles and applications. IEEE Journal of Quantum Electronics 26, 2186-2199. 
2. Kienle, A., C. Wetzel, A. Bassi, D. Comelli, P. Taroni, and A. Pifferi (2007) Determination of the optical properties of anisotropic biological media using an isotropic diffusion model. J. Biomed. Opt. 12, 014026-1-014026-9.

3. Cheng, W.-T., M.-T. Liu, H.-N. Liu, and S.-Y. Lin (2005) Micro-Raman spectroscopy used to identify and grade human skin pilomatrixoma. Microsc. Res. Tech. 68, 75-79.

4. Caspers, P. J., G. W. Lucassen, and G. J. Puppels (2003) Combined in vivo confocal Raman spectroscopy and confocal microscopy of human skin. Biophys. J. 85, 572-580.

5. Dam, J. S. (2001) Optical analysis of biological media - continuous wave techniques. $\mathrm{PhD}$ Thesis, Lund Institute of Technology.

6. Pickering, J., S. Prahl, N. Van Wieringen, J. Beek, H. Sterenborg, and M. van Gemert (1993) Double-integrating-sphere system for measuring the optical properties of tissue. Appl. Opt. 32, 399-410.

7. Bashkatov, A. N., E. A. Genina, V. I. Kochubey, and V. V. Tuchin (2005) Optical properties of human skin, subcutaneous and mucous tissues in the wavelength range from 400 to $2000 \mathrm{~nm}$. Journal of Physics D: Applied Physics 38, 2543-2555.

8. Roggan, A., M. Friebel, K. Dörschel, A. Hahn, and G. Müller (1999) Optical properties of circulating human blood in the wavelength range 400-2500 nm. J. Biomed. Opt. 4, $36-46$.

9. Salomatina, E., B. Jiang, J. Novak, and A. N. Yaroslavsky (2006) Optical properties of normal and cancerous human skin in the visible and near-infrared spectral range. $J$. Biomed. Opt. 11.

10. Beek, J. F., P. Blokland, P. Posthumus, M. Aalders, J. W. Pickering, H. J. C. M. Sterenborg, and M. J. C. Van Gemert (1997) In vitro double-integrating-sphere optical properties of tissues between 630 and 1064 nm. Phys. Med. Biol. 42, 2255-2261.

11. Van Gemert, M. J. C., S. L. Jacques, H. J. C. M. Sterenborg, and W. M. Star (1989) Skin optics. IEEE Trans. Biomed. Eng. 36, 1146-1154.

12. Meinhardt, M., R. Krebs, A. Anders, U. Heinrich, and H. Tronnier (2009) Absorption spectra of human skin in vivo in the ultraviolet wavelength range measured by optoacoustics. Photochemistry and Photobiology 85, 70-77.

13. Meinhardt, M., R. Krebs, A. Anders, U. Heinrich, and H. Tronnier (2008) Effect of ultraviolet adaptation on the ultraviolet absorption spectra of human skin in vivo. Photodermatology Photoimmunology and Photomedicine 24, 76-82.

14. Masuda, Y., T. Yamashita, T. Hirao, and M. Takahashi (2009) An innovative method to measure skin pigmentation. Skin Research and Technology 15, 224-229.

15. Doornbos, R. M. P., R. Lang, M. C. Aalders, F. W. Cross, and H. J. C. M. Sterenborg (1999) The determination of in vivo human tissue optical properties and absolute chromophore concentrations using spatially resolved steady-state diffuse reflectance spectroscopy. Phys. Med. Biol. 44, 967-981. 
16. Karsten, A. E. (2008) Effect of Wavelength, Epidermal Thickness and Skin Type on the Required Dose for Photodynamic Therapy. In Proceedings of the International Conference of the World Association of Laser Therapy, (Edited by Abrahanse, H.), pp. 137-143. Monduzzi Editore, Modemond S.r.l., Bologna, Italy, International Conference of the World Association of Laser Therapy, Sun City, South Africa, 19 October 2008.

17. Marieb, E. N. (2004) Human anatomy and physiology. Pearson Benjamin Cummings, San Francisco, California.

18. Masukawa, Y., H. Narita, E. Shimizu, N. Kondo, Y. Sugai, T. Oba, R. Homma, J. Ishikawa, Y. Takagi, T. Kitahara, Y. Takema, and K. Kita (2008) Characterization of overall ceramide species in human stratum corneum. J. Lipid. Res. 49, 1466-1476.

19. Young, A. R. (1997) Chromophores in human skin. Phys. Med. Biol. 42, 789-802.

20. McGinness, J. and P. Proctor (1973) The importance of the fact that melanin is black. Journal of Theoretical Biology 39, 677-678.

21. Urbanska, K., B. Romanowska-Dixon, Z. Matuszak, J. Oszajca, P. Nowak-Sliwinska, and G. Stochel (2002) Indocyanine green as a prospective sensitizer for photodynamic therapy of melanomas. Acta Biochimica Polonica 49, 387-391.

22. Alaluf, S., S. Alaluf, A. Heath, N. I. K. Carter, D. Atkins, H. Mahalingam, K. Barrett, R. I. A. Kolb, and N. Smit (2001) Variation in melanin content and composition in type $\mathrm{V}$ and VI photoexposed and photoprotected human skin: The dominant role of DHI. Pigment Cell Research 14, 337-347.

23. Alaluf, S., D. Atkins, K. Barrett, M. Blount, N. Carter, and A. Heath (2002) Ethnic variation in melanin content and composition in photoexposed and photoprotected human skin. Pigment Cell Research 15, 112-118.

24. Alaluf, S., D. Atkins, K. Barrett, M. Blount, N. Carter, and A. Heath (2002) The impact of epidermal melanin on objective measurements of human skin colour. Pigment Cell Research 15, 119-126.

25. Bashkatov, A. N., E. A. Genina, V. I. Kochubey, M. M. Stolnitz, T. A. Bashkatova, O. V. Novikova, A. Y. Peshkova, and V. V. Tuchin (2000) Optical properties of melanin in the skin and skin-like phantoms. In Proceedings of SPIE - The International Society for Optical Engineering, Vol. 4162, pp. 219-226.

26. Michels, R., F. Foschum, and A. Kienle (2008) Optical properties of fat emulsions. Opt. Exp. 16, 5907-5925.

27. Shimada, M., Y. Yamada, M. Itoh, and T. Yatagai (2001) Melanin and blood concentration in a human skin model studied by multiple regression analysis: Assessment by Monte Carlo simulation. Phys. Med. Biol. 46, 2397-2406.

28. Campbell, M. K. (2006) Biochemistry. Thomson Learning, Pacific Grove, California; Brooks/Cole, London.

29. Matuszak, Z. and M. Wasilewska-Radwanska (2006) Optical properties of melanin solutions. Estimation of polymer particle size. In Proceedings of the Symposium of 
Photonics Technologies for 7th Framework Program, pp. 533-537. Symposium of Photonics Technologies for $7^{\text {th }}$ Framework Program, Wroclaw, Poland, 12 October 20060.

30. Grobler, A. F., A. F. Kotzé, and J. du Plessis (2008) The design of a skin-friendly carrier for cosmetic compounds using Pheroid ${ }^{\mathrm{TM}}$ technology. In Science and Applications of Skin Delivery Systems. (Edited by J. W. Wiechers), pp. 283-311. Allured Publishing Corporation, Illinois, USA.

31. Tuchin, V. V. (2007) Tissue Optics: Light Scattering Methods and Instruments for Medical Diagnostics. SPIE Press, Bellingham, Washington, USA.

32. Meredith, P., B. J. Powell, J. Riesz, S. P. Nighswander-Rempel, M. R. Pederson, and E. G. Moore (2006) Towards structure-property-function relationships for eumelanin. Soft Matter 2, 37-44.

33. Kollias, N. and A. H. Baqer (1988) The role of human melanin in providing photoprotection from solar mid-ultraviolet radiation $(280-320 \mathrm{~nm})$. Journal of the Society of Cosmetic Chemists 39, 347-354.

34. Jacques, S. L. and D. J. McAuliffe (1991) The melanosome: threshold temperature for explosive vaporization and internal absorption coefficient during pulsed laser irradiation. Photochemistry and Photobiology 53, 769-775.

35. Kollias, N. (1995) The physical basis of skin color and its evaluation. Clinics in Dermatology 13, 361-367.

36. Wan, S., R. R. Anderson, and J. A. Parrish (1981) Analytical modeling for the optical properties of the skin with in vitro and in vivo applications. Photochemistry and Photobiology 34, 493-499.

37. Nofsinger, J. B., E. E. Weinert, and J. D. Simon (2002) Establishing structure-function relationships for eumelanin. Biopolymers - Biospectroscopy Section 67, 302-305.

38. Flock, S. T., S. L. Jacques, B. C. Wilson, W. M. Star, and M. J. C. Van Gemert (1992) Optical properties of intralipid: A phantom medium for light propagation studies. Lasers in Surgery and Medicine 12, 510-519.

39. van Staveren, H. J., C. J. M. Moes, J. van Marie, S. A. Prahl, and M. J. C. Van Gemert (1991) Light scattering in Intralipid-10\% in the wavelength range of 400-1100 nm. Appl. Opt. 30, 4507-4514.

40. Costin, G. E. and V. J. Hearing (2007) Human skin pigmentation: Melanocytes modulate skin color in response to stress. FASEB Journal 21, 976-994. 


\section{FIGURE CAPTIONS}

Figure 1. (a) Schematic representation of human skin. (b) Skin model showing the different skin layers. Graphics reproduced with the permission of Dr. J.S. Dam (http://cv.jsdam.dk/docs/pubs/Dam2000phd_Optical\%20analysis\%20of\%20biological\%20media.pdf).

Figure 2. Attenuation coefficient as a function of (a) wavelength and (b) melanin concentration for synthetic eumelanin samples at $\mathrm{pH} \sim 7.01$.

Figure 3. Attenuation coefficient as a function of wavelength for skin-like phantoms containing (a) $3 \mu \mathrm{L}$ Pheroid $^{\mathrm{TM}}$, (b) $3 \mu \mathrm{L}$ Intralipid, (c) $30 \mu \mathrm{L}$ Pheroid $^{\mathrm{TM}}$ and (d) $30 \mu \mathrm{L}$ Intralipid. Phantoms are compared to the melanin only samples of same concentrations from Fig. 2(a).

Figure 4. Comparison attenuation spectra for increasing concentrations of Pheroid ${ }^{\mathrm{TM}}$ (grey lines) and Intralipid (black lines).

Figure 5. Attenuation coefficient as a function of (a) Pheroid ${ }^{\mathrm{TM}}$ and (b) Intralipid concentration over the wavelength range 400 to $900 \mathrm{~nm}$. 
Figure 6. Average of skin-like phantoms made up of 0.0088 to $0.066 \mathrm{mg} / \mathrm{mL}$ melanin concentrations with added (a) $3 \mu \mathrm{L}$ and (b) $30 \mu \mathrm{L}_{\text {Pheroid }}^{\mathrm{TM}}$ and Intralipid respectively. Corresponding melanin only concentrations were used as references / blanks. Error bars represent the standard deviation to the average. 